\title{
Dynamic processes of domain switching in lead zirconate titanate under cyclic mechanical loading by in situ neutron diffraction
}

\author{
Soodkhet Pojprapai(Imlao) ${ }^{\text {a,b,*}}{ }^{\text {, }}$ Zhenhua Luo ${ }^{a}$, Bjørn Clausen ${ }^{c}$, Sven C. Vogel ${ }^{c}$, \\ Donald W. Brown ${ }^{\mathrm{c}}$, Jennifer Russel ${ }^{\mathrm{a}}$, Mark Hoffman ${ }^{\mathrm{a}}$ \\ ${ }^{a}$ School of Materials Science and Engineering, UNSW, Sydney, NSW, Australia \\ ${ }^{\mathrm{b}}$ School of Ceramic Engineering, Suranaree University of Technology, Thailand \\ ${ }^{c}$ Los Alamos Neutron Science Center, Los Alamos National Laboratory, Los Alamos, NM, USA
}

Received 16 July 2009; received in revised form 24 October 2009; accepted 20 November 2009

Available online 13 January 2010

\begin{abstract}
The performance of ferroelectric ceramics is governed by the ability of domains to switch. A decrease in the switching ability can lead to degradation of the materials and failure of ferroelectric devices. In this work the dynamic properties of domain reorientation are studied. In situ time-of-flight neutron diffraction is used to probe the evolution of ferroelastic domain texture under mechanical cyclic loading in bulk lead zirconate titanate ceramics. The high sensitivity of neutron diffraction to lattice strain is exploited to precisely analyze the change of domain texture and strain through a full-pattern Rietveld method. These results are then used to construct a viscoelastic model, which explains the correlation between macroscopic phenomena (i.e. creep and recovered deformation) and microscopic dynamic behavior (i.e. ferroelastic switching, lattice strain).
\end{abstract}

(C) 2009 Acta Materialia Inc. Published by Elsevier Ltd. All rights reserved.

Keywords: Ferroelectric; Ferroelastic; Domain switching; Neutron diffraction

\section{Introduction}

Ferroelectric ceramics, including lead zirconate titanate (PZT), are commonly used in many sensor and actuator applications, including pressure sensors, acceleration sensors, ultrasonic motors and piezo linear motors. Ferroelectric materials have been used in these applications because of their ability to convert applied electrical energy to mechanical strain, and applied mechanical energy to electrical charge. This unique property is known as the "piezoelectric effect". Theoretically, the piezoelectric effect is caused by the relative movement of ions in the ferroelectric crystals due to electrical or mechanical energy which leads to a reoriented state of polarization in the crystals. In ferroelectric materials with a tetragonal phase, the polariza-

\footnotetext{
* Corresponding author. Address: School of Ceramic Engineering, Suranaree University of Technology, Thailand. Tel.: +66 44224542.

E-mail address: soodkhet@g.sut.ac.th (S. Pojprapai(Imlao)).
}

tion direction is always parallel to the longer $(c)$ axis of the unit cell. When crystals with the same polarization align, a domain is formed in ferroelectric materials. Domains that possess different polarization orientations are separated by domain walls. The domains in a tetragonal ferroelectric can be reoriented $180^{\circ}$ or $90^{\circ}$ from the original direction by an electric field; this is termed $180^{\circ}$ and $90^{\circ}$ domain switching, respectively. On the other hand, domains can be reoriented only $90^{\circ}$ by a mechanical stress, a so-called $90^{\circ}$ or ferroelastic switching. In most sensor and actuator devices, ferroelectric materials are prone to fatigue due to cyclic electrical or mechanical loading. The fatigue manifests its effect as a reduction in domain switching or domain wall movement and subsequent premature failure of the device.

In the case of ferroelastic switching in tetragonal ferroelectric materials, domains with the $c$-axis parallel to the loading direction are reoriented to the direction that was once the $a$ - or $b$-axis. The change of domain orientation 
$\left(90^{\circ}\right.$ domain switching) can be detected by quantifying the intensity changes of certain peaks in the diffraction pattern, e.g. the " $c$ " $\left(\begin{array}{lll}0 & 0 l\end{array}\right)$ and " $a$ " $\left(\begin{array}{lll}h & 0 & 0\end{array}\right)$ peaks in tetragonal perovskite ceramics [1-3]. To probe ferroelastic switching behavior of bulk ferroelectric ceramics, neutron diffraction is an excellent technique compared with conventional X-ray diffraction because it provides good grain statistics of a sample (i.e. there is a sufficient number of grains of the sample in the gauge volume of the diffracted neutron beam) due to the relatively low absorption and high penetration of neutrons. Recent works have provided valuable information on ferroelastic behavior [4-12]. However, these investigations did not specify dynamic behavior of ferroelastic switching. Knowledge of dynamic ferroelastic behavior is essential in order to develop a failure analysis model for fatigue behavior. Therefore, this work aims to explain the evolution of ferroelastic switching behavior under mechanical cyclic loading by employing two stateof-the-art time-of-flight (TOF) neutron diffractometers at the Manuel Lujan Jr. Neutron Scattering Center, LANSCE, Los Alamos National Laboratory, USA. The HIPPO (HIgh Pressure-Preferred Orientation) neutron diffractometer was used to probe the change of complete domain texture before and after mechanical loading ex situ. The SMARTS (Spectrometer for Materials Research at Temperature and Stress) neutron diffractometer was used to investigate the dynamic behavior of domain switching and lattice strain. The diffraction patterns obtained from the SMARTS diffractometer were collected in situ during cyclic loading and used to present the trend of domain orientations reflecting ferroelastic dynamic behavior. Moreover, a successful viscoelastic model was developed to explain the linkage between macroscopic (i.e. creep and recovered deformation), and microscopic behavior (i.e. lattice strain, domain switching) during cyclic loading.

\section{Materials and methods}

A commercial soft PZT sample with tetragonal phase was prepared by Thales Underwater Systems Pty Ltd. (Australia). The samples were cut into cylinders of $6.67 \mathrm{~mm}$ diameter and $20 \mathrm{~mm}$ length and poled along the axial direction at $24 \mathrm{kV}$ at $120^{\circ} \mathrm{C}$. The piezoelectric constant $\left(d_{33}\right)$ of the sample after poling was $595 \mathrm{pC} \mathrm{N}^{-1}$. All samples were rounded at the edges to prevent chipping and cracking during cyclic loading experiments.

In this experiment, two sub-procedures are used to detect domain-switching behavior due to mechanical loading. First, the HIPPO diffractometer, a very powerful instrument for bulk texture analysis, was used to investigate domain texture change ex situ before and after loading. Second, the SMARTS diffractometer was applied to detect dynamic domain-switching behavior in situ during cyclic loading. For this work, it has to be noted that these two diffractometers are designed for different purposes; HIPPO is an instrument for bulk texture analysis while SMART is suitable for in situ (ferroelastic) strain measurement. When combining the results from these two diffractometers, a complete analysis of domain-switching behavior can be achieved.

\subsection{Domain texture analysis using the HIPPO diffractometer}

The domain texture of a poled sample before being subjected to the fatigue testing, which is represented by 002 and 200 pole figures, was determined using the HIPPO diffractometer. Then, the sample was subjected to the cyclic loading under a stress of 8-80 MPa up to 3000 cycles. The change of bulk texture after cyclic testing was revealed and confirmed by the HIPPO diffractometer. In the case of a typical texture measurement using HIPPO, spectra are recorded from four rotation positions of a sample and 30 detectors are used: eight detectors at the diffraction angles of $2 \theta=140^{\circ}, 10$ detectors at $2 \theta=90^{\circ}$, and 12 detectors at $2 \theta=40^{\circ}$ (details of HIPPO have been published elsewhere [13]). The collection time of diffracted neutrons of each rotation was $60 \mathrm{~min}$, so the complete texture measurement for one sample required $240 \mathrm{~min}$.

\subsection{Dynamic domain switching analysis using the SMARTS diffractometer}

In this experiment, TOF neutron diffraction patterns were collected in situ while the sample was subjected to cyclic loading in the SMARTS neutron diffractometer. To conduct in situ testing, the sample was mounted on an Instron load frame installed in the diffractometer. SMARTS contains two banks of detectors, which consist of a total of 384 single-ended ${ }^{3} \mathrm{He}$ tubes. The detectors are fixed and aligned at $\pm 90^{\circ}$ to the incident beam. The load axis of the load frame was oriented at $45^{\circ}$ to the incident beam (Fig. 1).

The detectors at $+90^{\circ}$ (detector bank 1) and $-90^{\circ}$ (detector bank 2) simultaneously record diffraction data according to the diffraction vectors $Q_{1}$ (perpendicular to the load axis) and $Q_{2}$ (parallel to the load axis), respec-

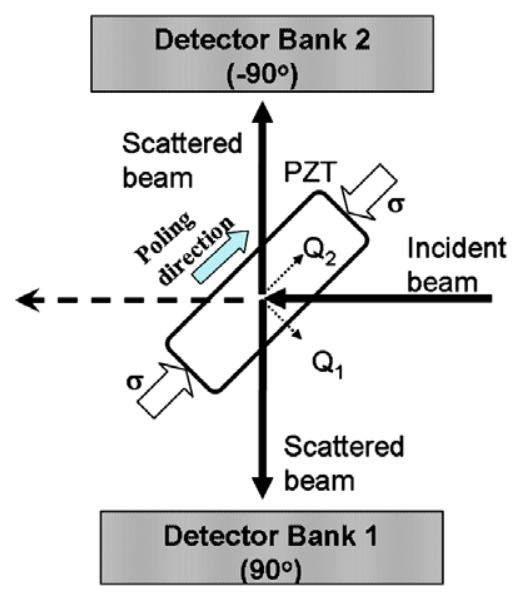

Fig. 1. The experiment geometry, showing the alignment of detectors, the sample, loading direction and neutron beams. 
tively. Details of the SMARTS diffractometer have been published elsewhere [14]. Before the experiment was conducted, the crosshead of the loading frame was painted with $\mathrm{GdO}_{2}$ to avoid diffraction interference from the crosshead material. In addition, the extensometer was shielded from the neutron beam by mounting a cadmium plate in front of it. To secure the sample and avoid domain switching while the sample was installed, the sample was fixed into the crosshead by preloading at $2 \mathrm{MPa}$. During the unloaded state of each cycle, the sample was held at a constant load of $8 \mathrm{MPa}$, which is well below the coercive stress required to induce domain switching (about $20 \mathrm{MPa}$ [4]). The cyclic loading test was conducted under load control, for which the maximum and minimum compressive stresses were 80 and $8 \mathrm{MPa}$, respectively, at a frequency of $0.1 \mathrm{~Hz}$. The macroscopic strain was measured using the extensometer. Domain-switching behavior can be detected by the intensity changes of $00 l$ and $h 00$ peaks obtained from the diffractometer. The quantitative analysis of domain switching is represented by the change in 002 pole density values reflecting the volume fraction of preferred $c$-domain orientation. Moreover, the change in lattice strains (microscopic strains) was extracted from diffraction patterns. During the test, the neutron diffraction patterns were collected at constant maximum $(80 \mathrm{MPa})$ and minimum $(8 \mathrm{MPa})$ stress following $1,10,100,300,500,10^{3}$ and $3 \times 10^{3}$ cycles. In this experiment, the term "loaded state" and "unloaded state" will represent the maximum (80 MPa) and minimum ( $8 \mathrm{MPa}$ ) stresses, which were held constant during diffraction data collection. To investigate dynamic ferroelastic behavior, the neutron spectra were collected at three intervals at each loading and unloaded state. A collection time of $300 \mathrm{~s}$ is required for each interval to provide a sufficient signal-to-noise ratio of the diffraction data.

\section{Data analysis}

In this experiment, the diffraction spectra obtained form the SMARTS and HIPPO diffractometers were analyzed via a full-pattern Rietveld analysis using the GSAS software package [15]. The space group of the PZT sample used in the Rietveld method was $P 4 \mathrm{~mm}$ for a tetragonal phase. The fitting parameters including the lattice parameters, background coefficients, histogram scale factor, diffractometer constants, absorption factors, reflectivity corrections and profile coefficients were initially refined. The initial lattice parameters of PZT samples were $a \cong 4.0429 \AA$, and $c \cong 4.1318 \AA$. These parameters are in agreement with prior work $[7,16,17]$. For domain global texture analysis, the spherical harmonic method, which is available in the GSAS software, was utilized; the spherical harmonic order $=2$ was assigned for the diffraction data collected from the HIPPO diffractometer. Because this present study focuses on the ferroelastic behavior under mechanical loading, the explanation of the refinement method will not be discussed. However, details of this method for powder diffraction analysis using the GSAS software can be found in prior work $[18,19]$. In addition to texture analysis, the diffraction data obtained from the SMARTS diffractometer were used to ascertain lattice strain, which is calculated from the difference between the lattice spacing before and after loading, expressed as:

$\varepsilon_{h k l}=\frac{d_{h k l}-d_{h k l}^{\mathrm{o}}}{d_{h k l}^{\mathrm{o}}}$,

where $\varepsilon_{h k l}$ is lattice strain, and $d_{h k l}$ and $d_{h k l}^{\circ}$ are the lattice spacings before and after loading. The lattice strain and lattice spacing are determined by a single peak fitting approach using a specific code (SMARTSware), which relies on GSAS, developed for the SMARTS diffractometer; the detail of the code is published elsewhere [20].

\section{Results}

\subsection{Change of global domain texture}

Before the results of ferroelastic dynamic behavior are determined, the general nature of the change of global domain texture before and after cyclic loading will be discussed. The complete domain texture of the poled sample can be achieved by employing the HIPPO diffractometer. These textures are represented by 002 and 200 pole figures which are extracted from the orientation distribution function (ODF) [21] calculated using the GSAS software package (Fig. 2a and b). 001 and 100 pole figures present the pole intensities of preferred $c$ - and $a$-domains, respectively. The pole intensity is normalized and presented in a unit of multiples of a random distribution (MRD) [22]; MRD values are indicated by colors shown in the color bar. In this case, MRD values correspond to the volume fraction of switched domains in the bulk sample. Theoretically, MRD of a 002 pole figure (or $\mathrm{MRD}_{00 l}$ ) $=3$ means that all $c$-domains orient along the poling direction. $\mathrm{MRD}_{00 l}=1$ means that $c$-domains are randomly oriented, while $\mathrm{MRD}_{00 l}=0$ means that all of the $c$-domains align perpendicular to the poling direction. In the case of the unloaded sample (Fig. 2a), the pole figure exhibits fiber texture due to electrical poling. The pole figure in Fig. 2a also indicates a texture of preferred $c$-domains along the axial direction of the sample which is exaggerated schematically in Fig. $2 a^{\prime}$. The change of the texture after fatigue testing is represented by the pole figure in Fig. 2b. In the latter case, the pole figure indicates that the sample possesses less preferred $c$-domain orientating along the axial direction (Fig. $2 b^{\prime}$ ). The decrease of the degree of preferred $c$-domain orientation is caused by $90^{\circ}$ domain switching induced by a compressive load which is known as mechanical depoling.

\subsection{Dynamic ferroelastic behavior}

To elucidate the dynamic mechanism of ferroelastic switching which leads to mechanical depoling, the diffraction spectra collected in situ during mechanical loading 


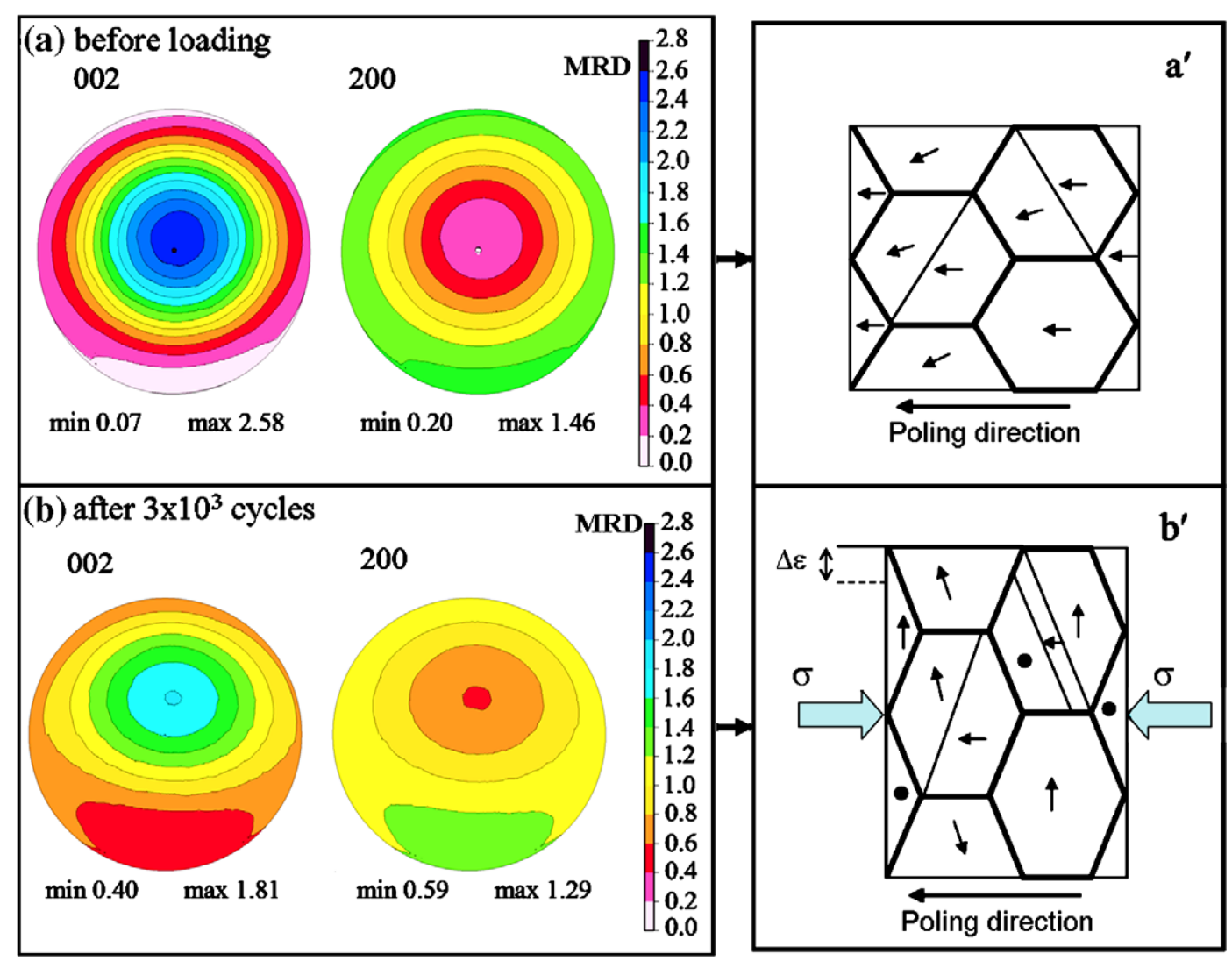

Fig. 2. The global texture of the poled sample which is probed by the HIPPO diffractometer: (a) before cyclic loading and (b) after cyclic loading. The visual aid to the possibility of domain alignment in sample $\left(\mathrm{a}^{\prime}\right)$, which processes preferred domain orientation, before cyclic loading, and sample $\left(\mathrm{b}^{\prime}\right)$, which is mechanically depoled, after 3000 cycles of loading is schematically shown beside the pole figures.

using the SMARTS diffractometer are analyzed. The neutron spectra obtained prior to mechanical loading are presented in Fig. 3 where detector bank 1 shows the diffraction data perpendicular to the load axis and detector bank 2 shows the diffraction parallel to the load axis. For this refinement, the $\chi$ factor, representing goodness of fit, is 1.598 which shows significant agreement between the Rietveld model and diffraction data [23].

The relative intensity ratio of the 002 peak to the 200 peak $\left(I_{002} / I_{200}\right)$ can be used to estimate the volume fraction of the c- and a-domains in the given sample direction. In Fig. 3, the small $I_{002} / I_{200}$ ratio from detector bank 1 suggests that, for this sample, there is a low volume fraction of $c$-domains oriented in the radial or transverse direction parallel to the scattering vector $Q_{1}$ (see Fig. 1). On the other hand, the $I_{002} / I_{200}$ ratio obtained from the detector bank 2 is much higher than that obtained from the detector bank 1 . These results confirm that there is a strongly preferred $c$-domain orientation along the poling, or sample axial, direction (parallel to the scattering vector $Q_{2}$ ).

In addition to the neutron diffraction data, the macroscopic axial strain during cyclic mechanical testing was recorded using the extensometer. The resultant strain behavior as a function of time is demonstrated in Fig. 4.
It can be seen that during periods of cyclic compression, cumulative compression strain occurred as observed in other configurations $[12,16]$. Furthermore, during $900 \mathrm{~s}$ holds at the maximum compressive stress $(80 \mathrm{MPa})$ compressive creep occurred, while $900 \mathrm{~s}$ holds at the minimum compressive stress $(8 \mathrm{MPa})$ were accompanied by strain recovery despite the sample still being subjected to a low compressive stress.

Creep and recovered deformation are much more pronounced in the first cycle. However, the time-dependent deformation decreases with increase in cycle number. During mechanical loading, the majority of creep and recovered deformation may be attributed to domain-switching behavior $[11,24,25]$. When the compressive load reaches the coercive stress, the $c$-domains, which are oriented along the axial direction, start switching to align as close as possible to the direction perpendicular to the compressive axis. On the other hand, when the load is removed, some domains switch back to the original direction. This ferroelastic switching process, including both forward and backward switching, involves the movement of ions in the crystal unit cell (i.e. the movement of $\mathrm{Ti}^{4+}, \mathrm{Zr}^{4+}$ ion relative to neighboring ions, $\left.\mathrm{Pb}^{2+}, \mathrm{O}^{2-}\right)$; the ions may be repelled or attracted by the same or different electrical charged ions [26-28], respectively. It has been proposed that the movement of ions in 

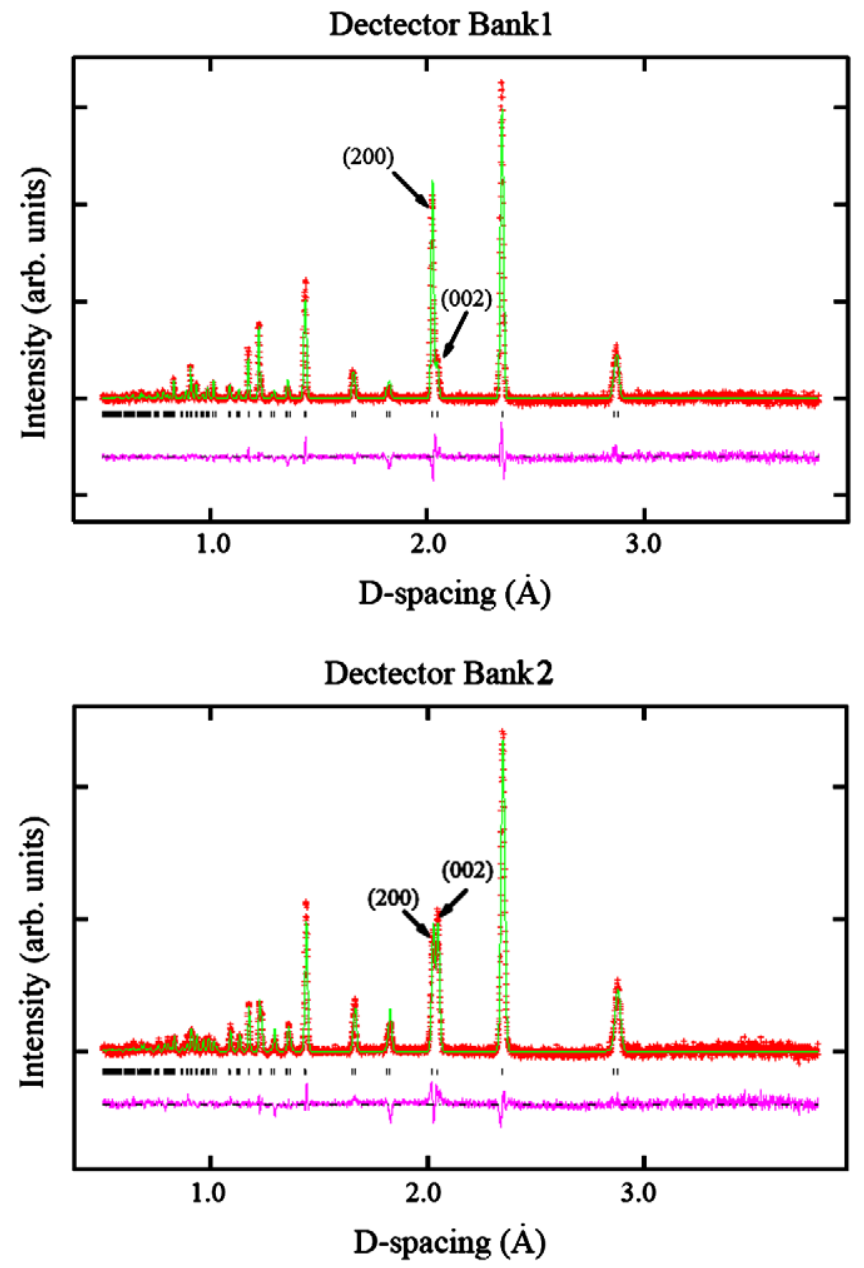

Fig. 3. The neutron diffraction patterns obtained from detector banks 1 and 2 before loading.

the unit cell during a switching process may be considered as the movement of particles in viscous media [29]. Therefore, this process involves a time-dependent mechanism that affects creep and recovery of the sample.

Using the GSAS software package, the diffraction data recorded during maximum and minimum loading were used to calculate the 002 pole intensity which reflects the volume fraction of switched $c$-domains in a unit of $\mathrm{MRD}_{00 l}$ as described in the previous section. In this section, the distribution orientation of preferred $c$-domains is presented by the distributions of $\mathrm{MRD}_{00 l}$ as a function of $\alpha$ angle, where $\alpha=0^{\circ}$ and $\alpha=90^{\circ}$ are the directions parallel and normal to the loading direction, respectively (see Fig. 5b1 for the sample and loading geometry). The distributions of $\mathrm{MRD}_{00 l}$ at different cycle number are presented in Figs. 5a1, 5b1 and $5 \mathrm{c} 1$. In this case, the values of $\mathrm{MRD}_{00 l}$ at $\alpha=0^{\circ}$ are considered because they are indicative of the change of volume fraction of $c$-domains in the axial direction which directly relates to the ferroelastic switching $\left(90^{\circ}\right.$ domain switching) in this direction. The lower the $\mathrm{MRD}_{00 l}$ values at $\alpha=0^{\circ}$, the smaller the volume fraction of $c$-domain in the axial direction.
Significantly, under the loaded state at the peak of the first cycle (i.e. the load is held at the maximum stress of $80 \mathrm{MPa}$ for $900 \mathrm{~s}$ ), the values of $\mathrm{MRD}_{00 l}$ decrease with time (Fig. 5a1). This result implies that the forward-switching process continuously takes place although the stress is kept constant. On the other hand, during the unloaded state at the end of the first cycle (i.e. at the minimum stress of $8 \mathrm{MPa}$ for $900 \mathrm{~s}$ ) $\mathrm{MRD}_{00 l}$ values increase with time (Fig. 5a1). This may be interpreted as showing that the forward-switched domains are recovered when the load is decreased to a lower level. This recovery process can be attributed to the process of backward-switching of domains. The evolution of domain-switching behavior through mechanical loading cycles is presented in Figs. $5 \mathrm{a} 1-5 \mathrm{c} 1$. From these figures, it can be seen that during the loaded state (a constant load of $80 \mathrm{MPa}$ ), creep deformation occurs in the bulk material at 1 and 10 cycles and the $\mathrm{MRD}_{00 l}$ values decrease with increasing time. However, this time dependence of domain switching under load is less significant following higher numbers of loading cycles (i.e. $3 \times 10^{3}$ cycles). Conversely, deformation recovery in the bulk sample and $\mathrm{MRD}_{00 l}$ values increase with time in the unloaded state (a constant load of $8 \mathrm{MPa}$ ). This recovery behavior is clearly detected following lower numbers of loading cycles (i.e. 1, 10 and 100 cycles), while is it is barely discernible after higher numbers of loading cycles (i.e. $10^{3}$, $3 \times 10^{3}$ cycles). These results show that the ability of domains to switch (including forward and backward switching) declines with the number of mechanical loading cycles. The decrease in domain switchability also reflects the effect of a cyclic fatigue environment on ferroelastic behavior.

From the change of $\mathrm{MRD}_{00 l}$ under cyclic loading, the microscopic strain represented by $c$-lattice strain is calculated by using SMARTSware. The results are shown in Figs. 5a2, $5 \mathrm{~b} 2$ and $5 \mathrm{c} 2$ following 1,10 and $3 \times 10^{3}$ loading cycles, respectively. In this experiment, the change of microstrain is represented by the change in $c$-lattice strain since it directly reflects ferroelastic strain in PZT ceramics with tetragonal phase [16]. It should be noted that all lattice strain measurements are referred to the lattice strain of the poled sample before applying compressive stress (as described in Eq. (1)). The trend of the time-dependent deformation of the lattice strain is nearly the same as that of domain switching. Similar to $\mathrm{MRD}_{00 l}$ values, creep and recovered deformation of the lattice strain can be observed following small numbers of loading cycles (i.e. 1, 10, 100 cycles). These phenomena almost disappear following higher numbers of loading cycles (i.e. $10^{3}, 3 \times 10^{3}$ cycles).

When considering the results from $\mathrm{MRD}_{00 l}$, lattice strain and macroscopic strain together, it can be seen that these results show a similar trend for creep and recovered deformation (see Figs. 4 and 6). In addition to the time dependence shown above, the relationship between macroscopic and microscopic behavior can be interpreted using the change of reversible strains and reversible domain switching during the loaded and unloaded state. To elucidate this relationship, the fraction of the reversible strains 

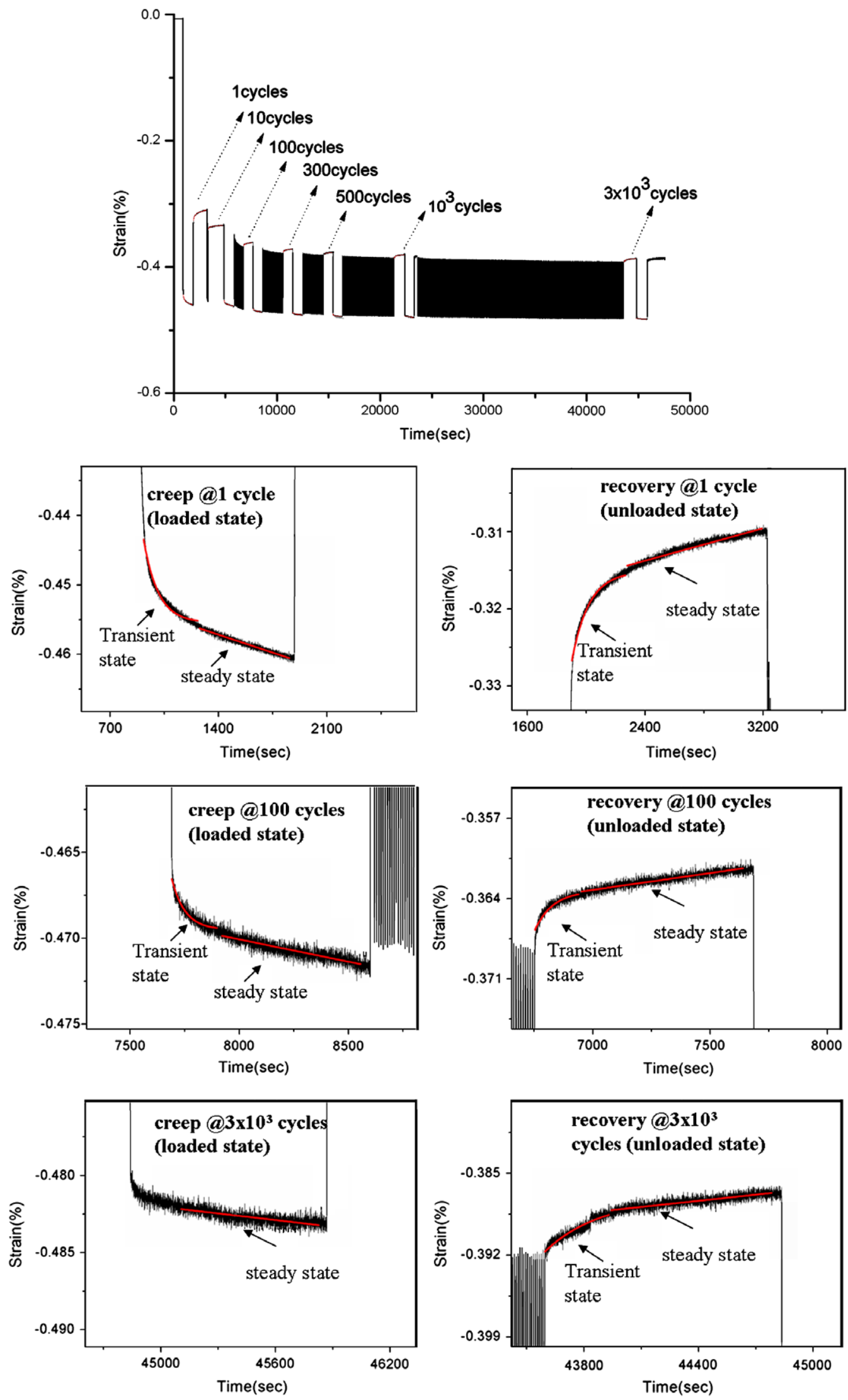

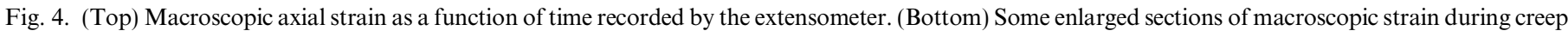

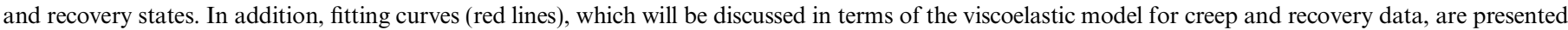

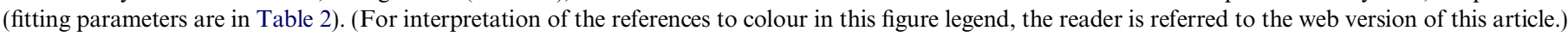



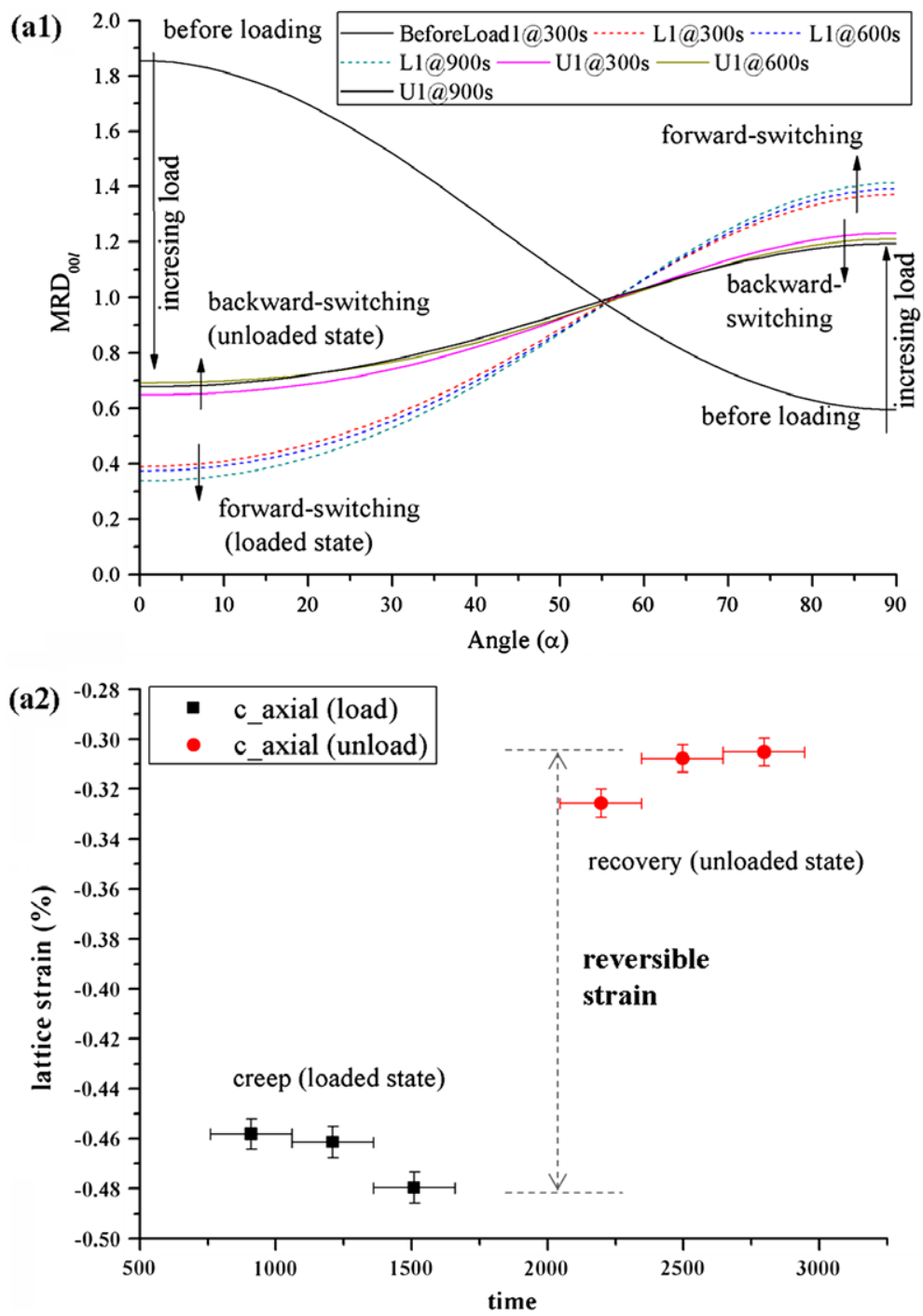

Fig. 5a. (a1) The evolution of $\mathrm{MRD}_{00 l}$ of $c$-domain as a function of orientation angle, $\alpha$, at 1 cycle; (a2) lattice strain as a function of time. In addition, (a2) shows the degree of reversible strain represented by the difference in strain at loading and in the unloaded state. A vertical bar of each datum shows the error of calculated strain while a horizontal bar shows collection time $(300 \mathrm{~s})$ during the loaded and unloaded states for each datum.

and the switchable domains during the stress was kept constant at $1,10,100,300,500,10^{3}$ and $3 \times 10^{3}$ cycles and is calculated using following equations:

$\varepsilon_{\text {mac }}^{\prime}=\varepsilon_{1, \mathrm{mac}}-\varepsilon_{\mathrm{ul}, \mathrm{mac}}$,

where $\varepsilon_{\text {mac }}^{\prime}, \varepsilon_{1, \text { mac }}$, and $\varepsilon_{\mathrm{ul}, \mathrm{mac}}$ are the reversible macroscopic strain, strain at the loaded state and strain at the unloaded state, respectively. The macroscopic strain was detected by the extensometer:

$\varepsilon_{\text {mic }}^{\prime}=\varepsilon_{1, \text { mic }}-\varepsilon_{\mathrm{ul}, \text { mic }}$,

where $\varepsilon_{\text {mic }}^{\prime}, \varepsilon_{1, \text { mic }}$, and $\varepsilon_{\mathrm{ul} \text {,mic }}$ are the reversible lattice strain, lattice strain at the loaded state and lattice strain at the unloaded state, respectively. The lattice strain was calculated using Eq. (1).

$\%$ Reversible domain $(\mathrm{RD})=\% \mathrm{BD}-\% \mathrm{FD}$,

where
$\% \mathrm{BD}(\%$ Backward switched domain $)=\frac{\mathrm{MRD}_{\text {unload }}}{\mathrm{MRD}_{\text {poled }}} \times 100$,

$\%$ FD $(\%$ Forward switched domain $)=\frac{M^{2} D_{\text {load }}}{M D_{\text {poled }}} \times 100$,

where $\mathrm{MRD}_{\text {poled }}, \mathrm{MRD}_{\text {load }}, \mathrm{MRD}_{\text {unload}}$, are the values of $\mathrm{MRD}_{00 l}$ before loading, during the loaded state and in the unloaded state, respectively. All MRD values are obtained from pole densities at $\alpha=0^{\circ}$.

By applying Eqs. (2)-(6), the reversible macro and lattice strains and the fraction of reversible domains as a function of the number of cycles are obtained, as shown in Fig. 6; errors for macroscopic strain data are shown in Table 1.

Significantly, the trends of these three sets of results are nearly the same. The percentage of reversible macroscopic 

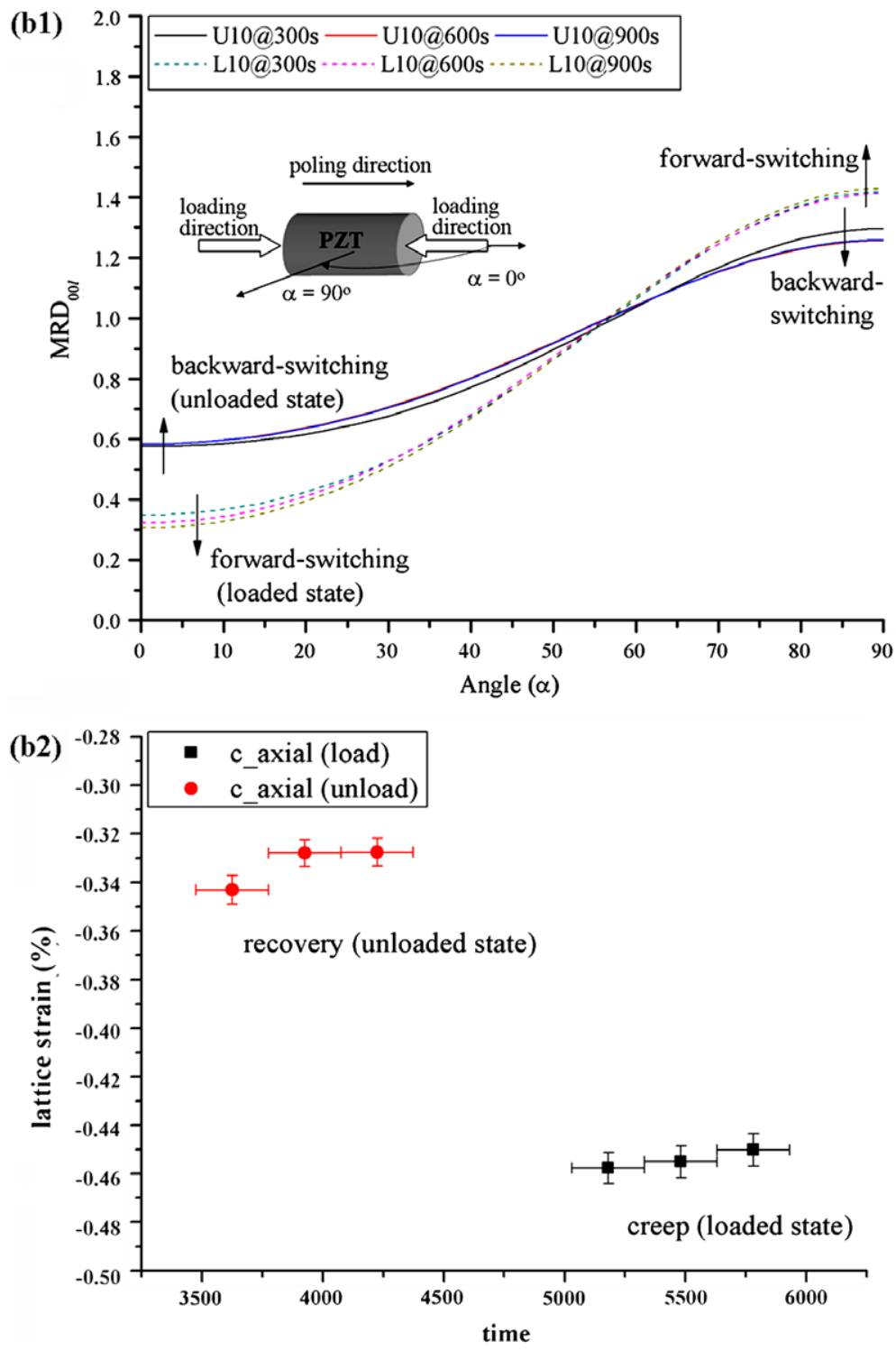

Fig. 5b. The evolution of: (b1) $\mathrm{MRD}_{00 l}$ and (b2) lattice strain at 10 cycles.

strain, reversible domain and lattice strain decreases exponentially with increase in the number of cycles. The reversible macroscopic and lattice strain becomes saturated after about 500 cycles, while the reversible domain seems to continuously decrease with number of cycles. These phenomena are in good agreement with our previous work $[16,30]$.

In this section, the correlation between macroscopic and microscopic behavior has been presented and quantitatively analyzed. To explain the dynamic mechanism of ferroelastic switching, a viscoelastic deformation model is proposed next.

\subsection{Viscoelastic model}

From the results shown in the previous subsection, when the compressive load was kept constant in the loaded or unloaded state, time-dependent deformation, including creep and recovery, was observed. The creep or recovery of the sample may be attributed to domain switching. The switching behavior in the loaded and unloaded state can therefore be explained by viscoelastic-based concepts [31-33].

With the viscoelastic-based concept, it may be assumed that there are three main component forces involved in the domain-switching mechanism under mechanical loading. The first component is the local mechanical force attributed to the applied load. This local force drives domains to orient at $90^{\circ}$ to the direction of the applied load. The second component is the viscous force represented by internal friction $\left(F_{\mathrm{f}}\right)$. For ferroelectric/ferroelastic ceramics, the internal friction may be caused by the interaction between the point defects which possess an electrical dipole, and domains which have spontaneous polarization [34-36]. 

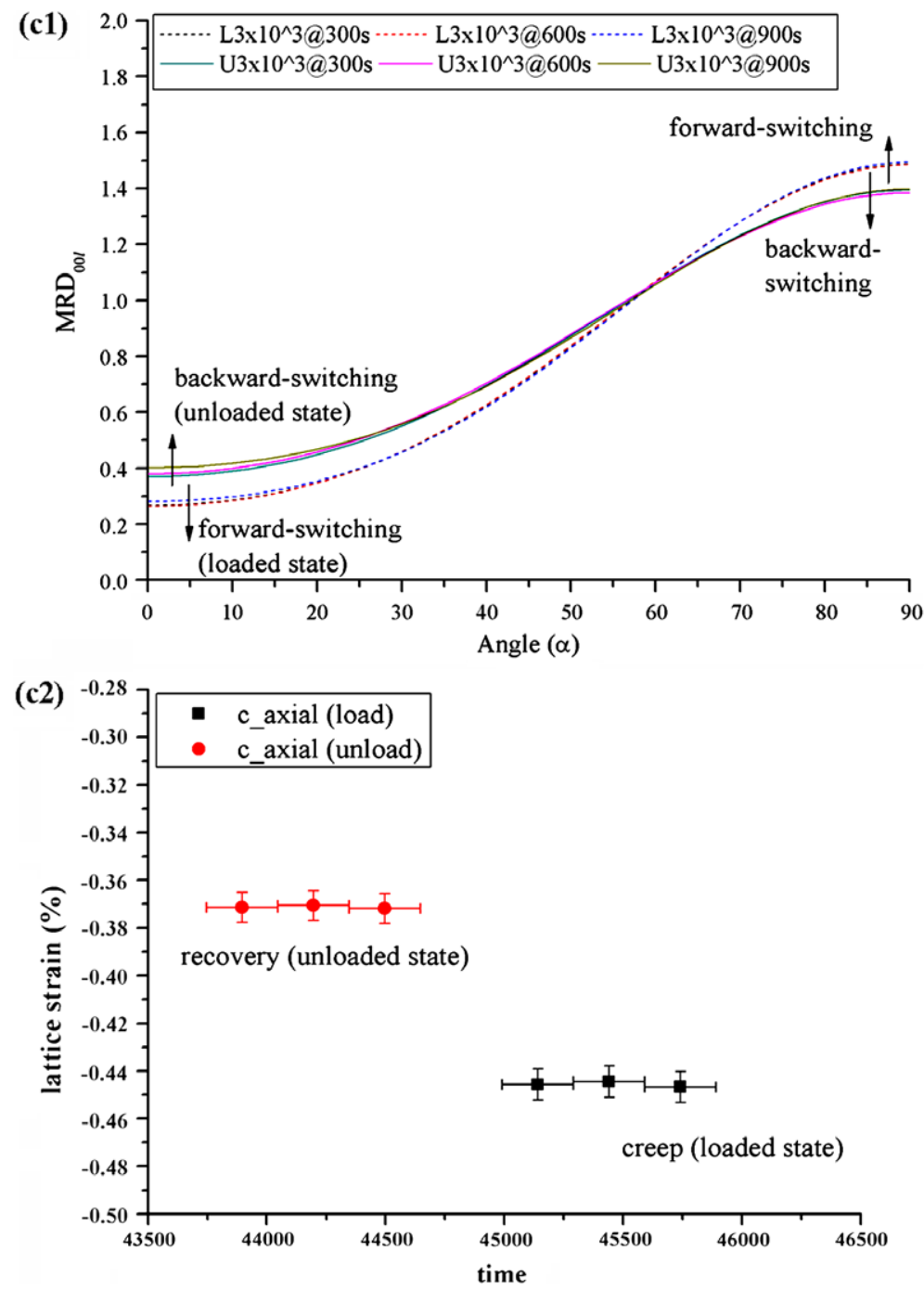

Fig. 5c. The evolution of: (c1) $\mathrm{MRD}_{00 l}$ and (c2) lattice strain at $3 \times 10^{3}$ cycles.

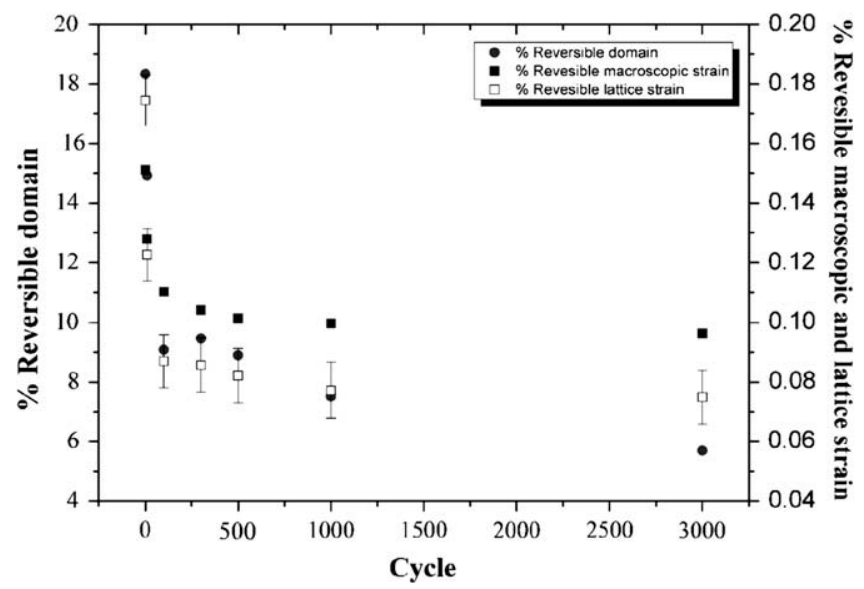

Fig. 6. The percentage of reversible domains and strains as a function of the number of cycles. The error bar of macroscopic strain is less than each datum, so errors of macroscopic data are shown in Table 1. The reversible domain datum is a single point obtained from the intersection of MRD function at $\alpha=0^{\circ}$ (Figs. 5a1, 5b1, and 5c1) so the error bar is not presented.
Table 1

Percent reversible macroscopic strain statistical errors.

\begin{tabular}{ll}
\hline$\%$ Reversible macroscopic strain & $\%$ Error \\
\hline 0.147723 & 0.002113 \\
0.126932 & 0.001794 \\
0.109467 & 0.001602 \\
0.104036 & 0.001413 \\
0.101214 & 0.0013 \\
0.099025 & 0.001454 \\
0.096072 & 0.001296 \\
\hline
\end{tabular}

This may lead to a decrease in domain switchability under cyclic loading. The last component is the restoring force $\left(F_{\mathrm{r}}\right)$, which drives a stabilization of the energy of the domain configuration. Therefore, this force will reorient the domains back to the stable configuration [33] via a backward-switching process. From this concept, the switching process entails the movement of ions in the unit cells. The movement of ions during a switching process 


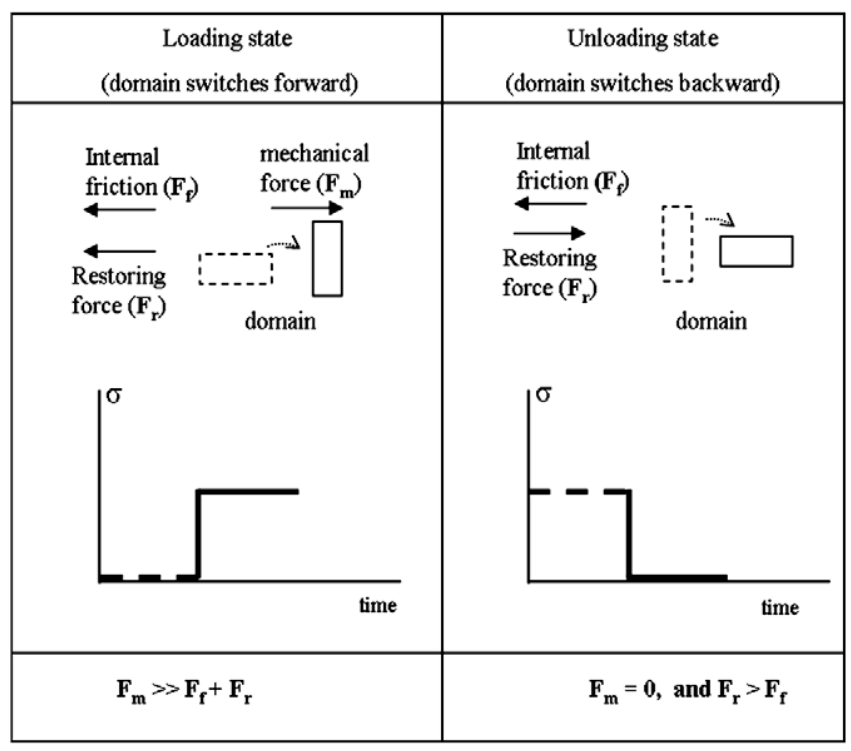

Fig. 7. A qualitative model used to explain forward- and backwardswitching mechanism based on a viscoelasticity concept [29].

may be considered as the movement of particles in viscous media; therefore, ferroelastic switching is a time-dependent mechanism which is reflected in the deformation of the bulk material. The influence of the various forces outlined above on the domain structure is illustrated in Fig. 7.

Here, we assume that the applied load is a square wave. In the case of the loaded state, when the mechanical load is applied and reaches the coercive stress, the local mechanical force overcomes the internal friction $\left(F_{\mathrm{f}}\right)$ and the restoring force $\left(F_{\mathrm{r}}\right)$. Consequently, the forward-switching process starts and domain wall movement occurs. Since this mechanism is time dependent, the domains continuously switch, although the load is held constant, leading to the bulk creep. On the other hand, after the load is removed $\left(F_{\mathrm{m}}=0\right)$, the backward-switching process starts if the friction force is less than the restoring force $\left(F_{\mathrm{f}}<F_{\mathrm{r}}\right)$. This causes bulk recovery deformation. Moreover, these reversible domains result in reversible ferroelastic strain. However, if the friction force is greater than the restoring force $\left(F_{\mathrm{f}}>F_{\mathrm{r}}\right)$, the domains do not switch back; hence, the domain walls do not move back to the original position. This may cause permanent or irreversible ferroelastic strain.

From a macroscopic view, the time-dependent deformation under mechanical loading of ferroelectric/ferroelastic ceramics can be explained by the schematic diagram shown in Fig. 8.

From Fig. 8, under the loaded state (0-1-2), there are three components of strain corresponding to the applied load. First, the elastic strain, $\varepsilon_{\mathrm{e}}$, is caused by stretching of ionic bonds in this material. This strain component instantly takes place at low applied stress and is reversible when the load is removed. Second, the transient creep strain, $\varepsilon_{\mathrm{ts}, \mathrm{c}}$, occurs immediately after the stress is held constant. In this state, the rate of deformation changes with
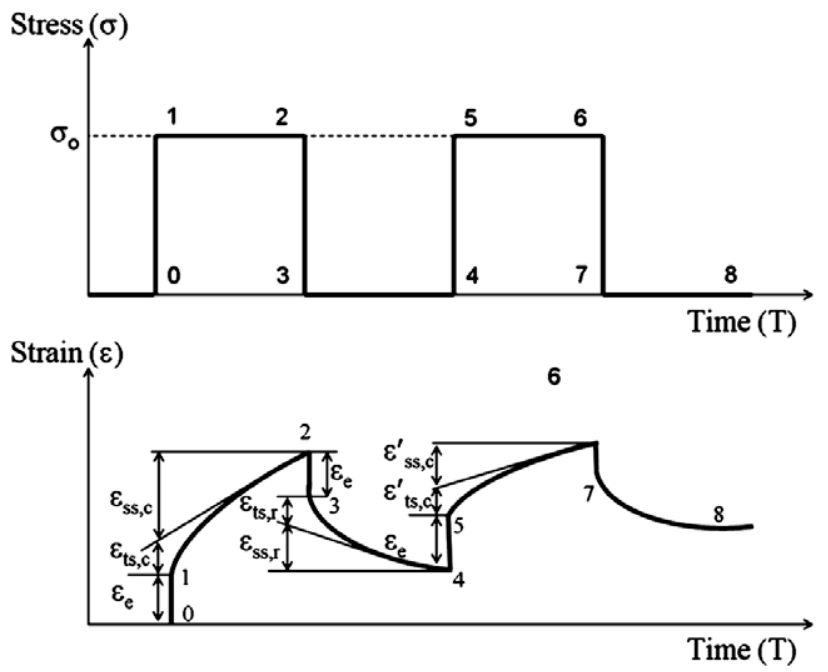

Fig. 8. The macroscopic time-dependent behavior under the cyclic loading. Subscripts ss is steady state, ts is transient state, e is elastic behavior, $\mathrm{c}$ is creep, and $\mathrm{r}$ is recovery.

the loading time. Theoretically, after the load is removed, most of this transient strain is reversed, which corresponds to reversible domains. This infers that the majority of the transient creep strain is caused by reversible domains. Finally, the steady-state creep strain, $\varepsilon_{\mathrm{ss}, \mathrm{c}}$, will replace the transient creep strain after a certain period of loading time. This strain component is not reversible and may be attributed to irreversible domains. Unlike creep deformation during the loaded state, the recovered strains are caused by the recovery mechanism involved in the domain backswitching process. At the unloaded state (2-3-4), when the load is removed or lowered below the coercive stress, recovery deformation occurs. The elastic strain, $\varepsilon_{\mathrm{e}}$, is recovered instantly. Then, the strain is recovered gradually with time and represented by the transient recovered strain, $\varepsilon_{\mathrm{ts}, \mathrm{r}}$. Eventually, the steady-state recovery strain, $\varepsilon_{\mathrm{ss}, \mathrm{r}}$, takes place during the unloaded state.

To quantify and explain the link between the macroscopic mechanism (time-dependent deformation) and the microscopic mechanism (domain switching), a rheological model is proposed (Fig. 9).

The model is composed of a combined Kelvin model (dashpot and spring in parallel) in series with a Maxwell model (dashpot and spring in series). This model can represent both creep and recovery behavior. A spring with the elastic constant $E_{1}$ (units of $\mathrm{N} \mathrm{m}^{-2}$ ) represents elastic behavior. A dashpot with the viscoelastic coefficient $\eta_{1}$ (units of $\mathrm{N} \mathrm{s} \mathrm{m}^{-2}$ ) represents steady-state behavior. The combination of a spring with elastic constant $E_{2}$ and a dashpot with the viscoelastic coefficient $\eta_{2}$ represents transient behavior. According to the model, the relationship between stress and strain as a function of time can be explained by the following equations [37].

In the case of creep deformation, the elastic strain $\varepsilon_{\mathrm{e}}$, the transient creep strain, $\varepsilon_{\mathrm{ts}, \mathrm{c}}$, and the steady-state creep strain, $\varepsilon_{\mathrm{ss}, \mathrm{c}}, \mathrm{can}$ be expressed as: 


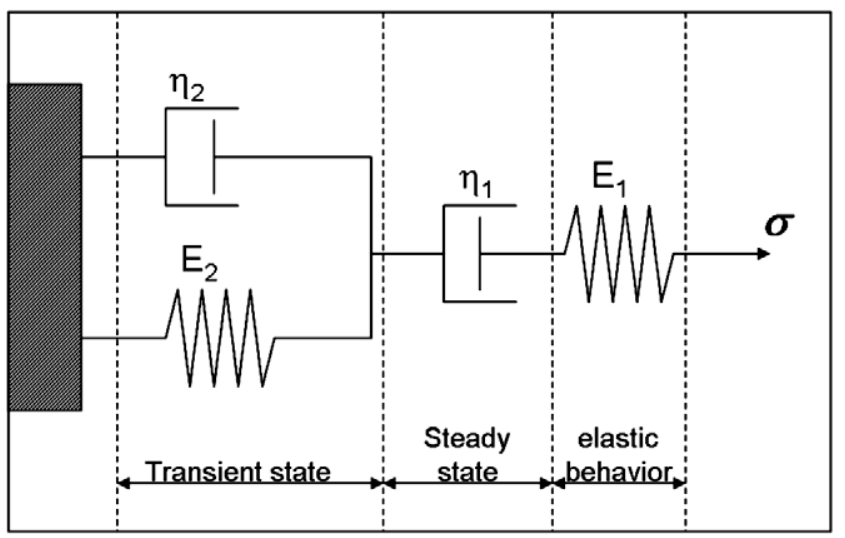

Fig. 9. A rheological model representing time-dependent deformation.

$\varepsilon_{\mathrm{e}, \mathrm{c}}=\frac{\sigma_{\mathrm{o}}}{E_{1}}$,

$\varepsilon_{\mathrm{ts}, \mathrm{c}}=\frac{\sigma_{\mathrm{o}}}{E_{2}}\left(1-e^{-t / \dot{\theta}}\right)$

$\varepsilon_{\mathrm{ss}, \mathrm{c}}=\frac{\sigma_{\mathrm{o}} \mathrm{t}}{\eta_{1}}$,

where the subscript $\mathrm{c}$ denotes the creep strain, $t$ is the time that a constant stress $\sigma_{\mathrm{o}}$ is maintained, and $\dot{\theta}=\frac{E_{2}}{\eta_{2}}$ with the units of time (s) where the retardation time reflects the duration of the transient creep deformation to approach completion.

In the case of recovery strain, the elastic strain represented by spring $E_{1}$ is recovered immediately after unloading, so this component is not calculated in this case. The steady-state strain is irreversible, so the dashpot $\eta_{1}$ component is included in this calculation. The transient recovered strain, $\varepsilon_{\mathrm{ts}, \mathrm{r}}$, and the steady-state recovered strain, $\varepsilon_{\mathrm{ss}, \mathrm{r}}$, can be expressed as:

$\varepsilon_{\mathrm{ts}, \mathrm{r}}=\varepsilon_{\mathrm{ts}, \mathrm{c}} e^{-\Delta t / \dot{\theta}}$

$\varepsilon_{\mathrm{ss}, \mathrm{r}}=\frac{\sigma_{\mathrm{o}} \mathrm{t}}{\eta_{1}}$,

where the subscript $\mathrm{r}$ denotes the recovered strain, $\Delta t$ is the time elapsed since the applied stress is removed, and $\dot{\theta}=\frac{E_{2}}{\eta_{2}}$ with the units of time (s) where the recovery time reflects the duration of the transient recovered strain to approach completion.

In this case, the important parameters used to characterize transient and steady- state behavior are $\dot{\theta}$ and $\frac{\sigma_{0}}{\eta_{1}}$, respectively. The parameter $\dot{\theta}$ indicates the duration of the transient strain which implies a time constant for the domain-switching process to stabilize (denoted as "transience time"). Moreover, $\dot{\theta}$ is related to the fraction of switchable domains which remain after cycling. For example, if $\dot{\theta}$ obtained from the first cycle is large, it may suggest that there are large numbers of switchable domains remaining at that loading cycle. The parameter $\frac{\sigma_{0}}{\eta_{1}}$ indicates the deformation rate, which is constant, during steady-state creep or recovery. This parameter also reflects the number of irreversible domains in a particular loading cycle. To characterize the creep and recovery behavior of the bulk sample, the transient and steady-state creep strain data (Fig. 4) are fitted to Eqs. (8) and (9), respectively. Furthermore, the transient and steady-state recovery strain data (Fig. 4) are fitted to Eqs. (10) and (11), respectively. Examples of fitted curves for transient and steady-state creep and recovery deformation are presented in Fig. 4. The fitting parameters $\theta$, and $\frac{\sigma_{0}}{\eta_{1}}$ are presented in Table 2.

Table 2 shows that, in the case of creep deformation, the transience time and creep rate decrease with the increase in the number of loading cycles. It is noted that the transience time at $10^{3}$ and $3 \times 10^{3}$ cycles cannot be calculated because the transient creep strain almost disappears. Decline of the transience time $(\dot{\theta})$ suggests that the time required for the domain-forward-switching process to stabilize becomes shorter. Moreover, the decrease of the creep rate $\left(\frac{\sigma_{0}}{\eta_{1}}\right)$ implies that the fraction of forward switchable domains decreases after a higher number of loading cycles. The same trend is also observed in the case of recovered deformation. However, the majority of recovered deformation is caused by the domain-back-switching process. The decrease of transience recovery time $(\dot{\theta})$ indicates that the average stabilization time for the backward-switching process decreases with an increase in number of loading cycles. Moreover, the decline in the recovery rate $\left(\frac{\sigma_{0}}{\eta_{1}}\right)$ may be caused by a decrease in the number of backward switchable domains. The significant decrease of forward and back-

Table 2

The fitting parameters $\dot{\theta}$, and $\frac{\sigma_{0}}{\eta_{1}}$ obtained from a strain vs. time curve (Fig. 4).

\begin{tabular}{|c|c|c|c|c|c|c|c|c|c|c|c|c|}
\hline \multirow[t]{3}{*}{ Cycle } & \multicolumn{6}{|c|}{ Creep behavior } & \multicolumn{6}{|c|}{ Recovery behavior } \\
\hline & \multicolumn{2}{|c|}{ Transient state } & & \multicolumn{2}{|l|}{ Steady state } & & \multicolumn{2}{|c|}{ Transient state } & & \multicolumn{2}{|l|}{ Steady state } & \\
\hline & $\begin{array}{l}\text { Trans. time, } \\
\dot{\theta}(\mathrm{s})\end{array}$ & $R^{2}$ & & $\begin{array}{l}\text { Creep rate, } \\
\sigma_{\mathrm{o}} / \eta\left(\mathrm{s}^{-1}\right)\end{array}$ & $\overline{R \text {-square }}$ & & $\begin{array}{l}\text { Trans. time, } \\
\dot{\theta}(\mathrm{s})\end{array}$ & $R^{2}$ & & $\begin{array}{l}\text { Recovery rate, } \\
\sigma_{\mathrm{o}} / \eta\left(\mathrm{s}^{-1}\right)\end{array}$ & $R$-square & \\
\hline 1 & 94.79 & 0.97 & Decrease $\Downarrow$ & $-7.40 \mathrm{E}-6$ & 0.96 & Decrease $\Downarrow$ & 115.86 & 0.98 & Decrease $\Downarrow$ & $5.25 \mathrm{E}-6$ & 0.93 & Decrease $\Downarrow$ \\
\hline 10 & 63.82 & 0.94 & & $-4.95 \mathrm{E}-6$ & 0.94 & & 85.10 & 0.89 & & $2.60 \mathrm{E}-6$ & 0.76 & \\
\hline 100 & 53.54 & 0.86 & & $-2.59 \mathrm{E}-6$ & 0.77 & & 77.90 & 0.88 & & $2.55 \mathrm{E}-6$ & 0.73 & \\
\hline 300 & 50.96 & 0.79 & & $-2.00 \mathrm{E}-6$ & 0.67 & & 76.80 & 0.88 & & $2.22 \mathrm{E}-6$ & 0.69 & \\
\hline 500 & 51.92 & 0.80 & & $-1.88 \mathrm{E}-6$ & 0.68 & & - & - & & $2.22 \mathrm{E}-6$ & 0.76 & \\
\hline 1000 & - & - & & $-1.80 \mathrm{E}-6$ & 0.59 & & - & - & & $2.10 \mathrm{E}-6$ & 0.75 & \\
\hline 3000 & - & - & & $-1.44 \mathrm{E}-6$ & 0.53 & & - & - & & $1.76 \mathrm{E}-6$ & 0.72 & \\
\hline
\end{tabular}


ward switchable domains may be attributed to the interaction between domain wall and point defects. Importantly, this switching behavior can be described by the viscoelastic model and based on the macroscopic data. Creep and recovered deformation obtained from macroscopic data correspond well with the switching behavior obtained from the neutron diffraction measurement.

\section{Conclusions}

In situ TOF neutron diffraction data may be used to probe the evolution of domain texture and the dynamic ferroelastic behavior under mechanical cyclic loading. The diffraction data are quantitatively analyzed by using a full-pattern Rietveld method via the GSAS package software. The change of domain global texture before and after loading is confirmed by the powerful texture analysis HIPPO diffractometer. The in situ measurement of dynamic ferroelastic mechanism of bulk PZT is achieved by employing the SMARTS diffractometer.

It is found that there is a strong link between timedependent deformation of the bulk PZT and ferroelastic switching. From the quantitative analysis, in the case of loading, the volume fraction of preferred $c$-domains represented by $\mathrm{MRD}_{00 l}$ values significantly decreases with the loading time during the loaded state. This is caused by the forward-switching process of $c$-domains. On the other hand, $\mathrm{MRD}_{00 l}$ values increase with time under the unloaded state because of the backward-switching process of $c$-domains. The trend of the change of $\mathrm{MRD}_{00 l}$ values with the time during the loaded or unloaded state is the same as that of the lattice strain. This evidence indicates that the strong correlation exists between macroscopic deformation (creep and recovered deformation) and a microstructural dynamic mechanism (a domain-forwardswitching or backward-switching process). Moreover, the results show that the degree of degradation of ferroelastic switching is more severe after the material has been subjected to a higher number of loading cycles. The mechanism of ferroelastic switching can be explained by using a viscoelastic model. Importantly, the model can be applied to describe the intimate relationship between macroscopic strain and ferroelastic domain switching.

\section{Acknowledgements}

Travel support to access these facilities was provided by the Access to Major Research Facilities (AMRF) program, administered by the Australian Nuclear Science and Technology Organisation (ANSTO), under proposal number $04 / 05-\mathrm{N}-23$. This work was financially supported by the ARC Discovery Grant No. DP0558596. S.P. thanks Dr. Jacob L. Jones, University of Florida, and Dr. Peter Bryant of Thales Underwater Systems Pty Ltd. (Australia) for technical assistance and fruitful discussions. This work has benefited from the use of the Lujan Neutron Scattering Center at LANSCE, funded by the
Department of Energy's Office of Basic Energy Sciences. Los Alamos National Laboratory is operated by Los Alamos National Security LLC under DOE Contract DE-AC52-06NA25396. This work was sponsored by the U.S. Dept. of Energy, Office of Basic Energy Sciences Project FWP 06SCPE401.

\section{References}

[1] Subbarao EC, McQuarrie MC, Buessem WR. J Appl Phys 1957;28:1194.

[2] Mehta K, Virkar AV. J Am Ceram Soc 1990;73:567.

[3] Jones JL, Slamovich EB, Bowman KJ. J Appl Phys 2005;97:034113.

[4] Cao H, Evans AG. J Am Ceram Soc 1993;76:890.

[5] Calderon-Moreno JM. Mater Sci Eng A 2001;315:227.

[6] Calderon-Moreno JM, Popa M. Mater Sci Eng A 2002;336:124.

[7] Rogan RC, Üstündag E, Clausen B, Daymond MR. J Appl Phys 2003;93:4104.

[8] Forrester JS, Kisi EH, Studer AJ. J Eur Ceram Soc 2005;25:447.

[9] Li JY, Rogan RC, Üstündag E, Bhattacharya K. Nat Mater 2005;4:776.

[10] Pojprapai S, Jones JL, Hoffman M, Vogel SC. J Am Ceram Soc 2006.

[11] Zhou D, Kamlah M. Acta Mater 2006;54:1389.

[12] Jones JL, Hoffman M, Vogel SC. Mech Mater 2007;39:283.

[13] Vogel SC, Hartig C, Lutterotti L, Von Dreele RB, Wenk HR, Williams DJ. Powder Diffr 2004;19:65.

[14] Bourke MAM, Dunand DC, Ustundag E. Appl Phys A: Mater Sci Process 2002;74:1707.

[15] Von Dreele RB, Jorgensen JD, Windsor CG. J Appl Cryst 1982;15:581.

[16] Pojprapai Imlao S, Jones JL, Hoffman M, Vogel SC. J Am Ceram Soc 2006;89:3567.

[17] Noheda B, Gonzalo JA, Cross LE, Guo R, Park SE, Cox DE, et al. Phys Rev B 2000;61:8687.

[18] Larson AC, Von Dreele RB. General structure analysis system (GSAS) LAUR 86-748, Los Alamos National Laboratory, NM; 2004.

[19] Toby B. J Appl Crystall 2001;34:210.

[20] Clausen B. SMARTSware manual. New Mexico, LA, NM: The Regents of the University of California; 2003.

[21] Bunge HJ. Texture analysis in material science: mathematical methods. London: Butterworths'; 1982.

[22] Kocks UF, Tomé CN, Wenk HR. Texture and anisotropy: preferred orientations in polycrystals and their effect on materials properties. Cambridge: Cambridge University Press; 1998.

[23] Young RA. The Rietveld method. New York: Oxford University Press; 1995.

[24] Guillon O, Thiébaud F, Delobelle P, Perreux D. J Eur Ceram Soc 2004;24:2547.

[25] Zhou D, Kamlah M. J Appl Phys 2005;98:104107.

[26] Jona F, Shirane G. Ferroelectric crystals. New York: Pergamon Press; 1962.

[27] Uchino K. Ferroelectric devices. New York: Marcel Dekker; 2000.

[28] Rabe KM, Ahn CH, Triscone JM, editors. Physics of ferroelectrics. Berlin: Springer Verlag; 2007.

[29] Pojprapai (Imlao) S, Jones LJ, Studer JA, Russell J, Valanoor N, Hoffman M. Acta Mater 2008.

[30] Pojprapai S, Jones JL, Studer AJ, Russell J, Valanoor N, Hoffman M. Acta Mater 2008.

[31] Fan J, Stoll WA, Lynch CS. Acta Mater 1999;47:4415.

[32] Harrison RJ, Redfern SAT. Phys Earth Planet Interiors 2002;134:253.

[33] Lente MH, Picinin A, Rino JP, Eiras JA. J Appl Phys 2004;95:2646.

[34] Robels U, Arlt G. J Appl Phys 1993;73:3454.

[35] Kim S, Gopalan V, Kitamura K, Furukawa Y. J Appl Phys 2001;90:2949.

[36] Kan Y, Lu X, Wu X, Zhu J. Appl Phys Lett 2006;89:262907.

[37] Dowling NE. Mechanical behavior of materials: engineering methods for deformation, fracture and fatigue. New Jersey: Prentice-Hall; 1999. 